\section{Olivier Goureau} Yves Courtois

\section{ADRESSE}

O. Goureau: chargé de recherche à l'Inserm. Y. Courtois: directeur de recherche à l'Inserm, directeur de l'unité 450 de l'Inserm. Inserm U. 450, développement, vieillissement et pathologie de la rétine, association Claude-Bernard, 29, rue Wilhem, 75016 Paris, France.

\section{TIRÉS À PART}

O. Goureau.

\title{
Monoxyde d'azote et rétine: un médiateur aux deux visages
}

Le monoxyde d'azote (NO) est produit à partir de la L-arginine, par l'enzyme NO synthase (NOS). Trois isoformes de NOS sont actuellement connues et codées par trois gènes distincts, NOS-I et NOS-III, dites isoformes constitutives, et la NOS de type II, communément appelée NOS inductible. Ces trois isoformes sont présentes dans la rétine et leur rôle commence à être éclairci. La production rapide et réglée de faibles quantités de NO par les formes I et III est responsable de certaines neurotransmissions et de la régulation du tonus artériolaire rétinien. En revanche, l'isoforme NOS-II est exprimée dans les cellules non neuronales de la rétine (cellules gliales et épithéliales), lors de certaines affections et après stimulation in vitro par des cytokines. Le NO produit en grande quantité par la NOS de type II est un agent bactéricide potentiel, mais peut également perturber le bon fonctionnement de la rétine, entraîner une inflammation de la rétine, voire même participer à certaines dégénérescences rétiniennes.

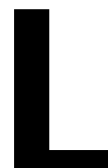

e monoxyde d'azote (NO) connu depuis longtemps comme un gaz toxique de l'environnement s'est révélé être un messager biologique particulièrement important. Une molécule aussi simple que NO est l'un des messagers les plus étudiés pour son implication dans un grand nombre de communications cellulaires liées aux grandes fonctions physiologiques du système vasculaire, de la neurobiologie et de l'immunologie $\left(m / s n^{\circ} 10\right.$, vol. 7, p. 1094). NO peut ainsi être, à la fois, un agent vasorelaxant, un messager neuronal intercellulaire et un agent bactéricide. Depuis cing ans, les ADNc et les gènes des enzymes responsables de la biosynthèse de NO, les NO synthases (NOS), ont été clonés et leurs produits bien caractérisés d'un point de vue biochimique (pour revues, voir: [1-4]). Il existe trois isoformes de NOS, NOS-I et NOS-III, dites isoformes constitutives, et les NOS de type II, communément appelées NOS inductibles*. Les premières, présentes principalement dans les neurones (NOS-I) et l'endothélium vas-

* NOS-I est aussi connue sous le nom de NOS neuronale et NOS-III sous celui de NOS endothéliale. La NOS-II, inductible, correspond à l'isoforme «macrophagique». 


\section{RÉFÉRENCES}

1. Bredt DS, Snyder SH. Nitric oxide : a physiologic messenger molecule. Annu Rev Biochem 1994; 63: 175-95.

2. Lacaze-Masmonteil T. Les monoxyde d'azote (NO) synthases. médecine/sciences $1992 ; 8$ : 843-5.

3. Nathan CF, Xie Q. Nitric oxide synthases: roles, tolls, and controls. Cell 1994; 78: 915-8.

4. Nussler AK, Billiar TR. Inflammation, immunoregulation, and inducible nitric oxide synthase. J Leukoc Biol 1993; 54: 171-8.

5. Moncada S, Higgs A. Mechanisms of disease. The L-arginine-nitric oxide pathway. N Engl J Med 1993; 329: 2002-12.

6. Crépel F, Lemaire G. Le monoxyde d'azote. médecine/sciences 1995; 11: 1639-42.

7. Venturini CM, Knowles RG, Palmer RMJ, Moncada S. Synthesis of nitric oxide in the bovine retina. Biochem Biophys Res Commun $1991 ; 180: 920-5$.

8. Yamamoto R, Bredt DS, Snyder SH, Stone RA. The localization of nitric oxide synthase in the eye and related cranial ganglia. Neuroscience 1993 ; 54 : 189-200.

9. Koch K, Lambrecht H, Haberecht M, Redburn D, Schimdt HHHW. Functional coupling of a calcium/calmodulin-dependent nitric oxide synthase and a soluble guanyl cyclase in vertebrate photoreceptor cells. EMBO J 1994; 13: 3312-20.

10. Perez MTR, Larsson B, Alm P, Andersson KE, Ehinger B. Localization of neuronal nitric oxide synthase-immunoreactivity in rat and rabbit retinas. Brain Res 1995; 104: 207-17.

11. Lieppe BA, Stone C, Koistinaho J, Copenhagen DR. Nitric oxide synthase in Mülfer cells and neurons of salamander and fish retina. J Neurosci 1994; 14: 7641-54.

12. Park C, Pardhasaradhi K, Gianotti C, Villegas E, Krishna G. Human retina expresses both constitutive and inducible isoforms of nitric oxide synthase mRNA. Biochem Biophys Res Commun 1994; 205 : 85-91.

13. Miyachi EI, Murakami M, Nakaki T. Arginine blocks gap junctions between retinal horizontal cells. Neuro Report 1990 ; 1: 10710.

14. Shiells R, Falk G. Retinal on bipolar cells contain a nitric oxide sensitive guanylate cy- culaire (NOS-III), produisent rapidement de faibles quantités de NO en réponse à une augmentation de la concentration de calcium intracellulaire. Cette libération de NO précisément réglée est responsable de certains phénomènes de neurotransmission et du tonus vasculaire basal. Les NOS de type II dont l'expression est réglée au niveau transcriptionnel par les cytokines et les endotoxines libèrent des quantités de NO beaucoup plus importantes et pendant des durées beaucoup plus longues (jours). Les mécanismes de régulation de l'activité de NOS-II sont très différents de ceux impliqués pour les NOS dites «constitutives». Les NOS de type II ont été décrites dans un grand nombre de cellules, telles que les macrophages, les hépatocytes, les astrocytes, les cellules musculaires lisses et les chondrocytes. Dans ce système inductible, NO revêt un caractère cytotoxique, en tuant des cellules cibles environnantes, via la perturbation de leur métabolisme (synthèse d'ADN, respiration, peroxydation membranaire...). Malheureusement NO s'attaque aussi bien aux bactéries étrangères qu'aux cellules saines avoisinant la source de NO, d'où sa nature très ambivalente, à la fois molécule efficace de défense contre les infections et responsable de certains désordres $\left(\mathrm{m} / \mathrm{s} n^{\circ} 12\right.$, vol. 11, p. 1743) [3-5].

$\mathrm{Au}$ cours des cinq dernières années, de nombreux travaux ont permis de caractériser les différentes formes de NOS ainsi que le rôle du NO dans le système nerveux central [6]. Si la plupart des études ont été effectuées dans le cerveau, la présence et le rôle du NO dans la rétine, ébauche terminale du système nerveux central, commencent à se dévoiler et les trois isoformes ont pu être identifiées (figure 1), par différentes techniques (NADPH-diaphorase, immunohistochimie, dosages enzymatiques, détection des messagers).

\section{Mise en évidence et rôle potentiel des différentes isoformes de la NOS dans la rétine}

\section{NOS constitutives (NOS-I, NOS-III)}

Une activité enzymatique de type NOS a pu être démontrée dans des extraits bruts de rétine et dans des segments externes isolés de photorécepteurs [7]. Les propriétés enzymatiques de cette enzyme et sa dépendance vis-à-vis du complexe calcium/calmoduline sont identiques à celles de la NOS-I présente dans le cerveau. Des études antérieures utilisant la technique de coloration à l'aide de la NADPH-diaphorase, qui reflète la présence d'une activité NOS, montraient un marquage dans la rétine de mammifère. Plus récemment, des études par immunohistochimie ont permis de localiser la NOS-I dans certaines cellules amacrines, dans des cellules horizontales et dans les photorécepteurs de différentes espèces [8-10], alors que la localisation de NOS-III par immunohistochimie n'a jamais été entreprise. Notons toutefois que le marquage obtenu par la NADPH-diaphorase dans les vaisseaux rétiniens et choroïdiens pourrait correspondre à l'isoforme III présente dans les cellules endothéliales. Concernant les autres cellules non neuronales de la rétine, la NOS-I a pu être mise en évidence par immunohistochimie dans les cellules gliales de Müller [11]. Les rares études de biologie moléculaire confirment que la rétine humaine exprime bien l'ARNm de NOS-I [12].

Le NO présent dans la rétine pourrait servir de neurotransmetteur dans la transmission synaptique rétinienne. En effet, plusieurs études montrent que des donneurs de NO sont capables de modifier le couplage électrique entre les cellules horizontales [13], d'activer des courants ioniques indirectement par une augmentation de GMPc dans les cellules bipolaires et dans les cellules ganglionnaires [14, 15] (figure 2). De plus NO est capable de moduler la transduction visuelle, puisqu'un donneur de NO de type nitrosocystéine est capable de moduler la conductance de certains canaux ioniques des photorécepteurs [16]. Des études électrophysiologiques sur des segments externes de photorécepteurs isolés montrent que la présence de NO modifie la dépolarisation subie par ces segments dans l'obscurité et altère leur réponse à la lumière [17]. Enfin, le rôle vasodilatateur du NO produit aussi bien par l'endothélium vasculaire en réponse à diverses stimulations (acétylcholine, histamine...) que par certaines terminaisons ner- 


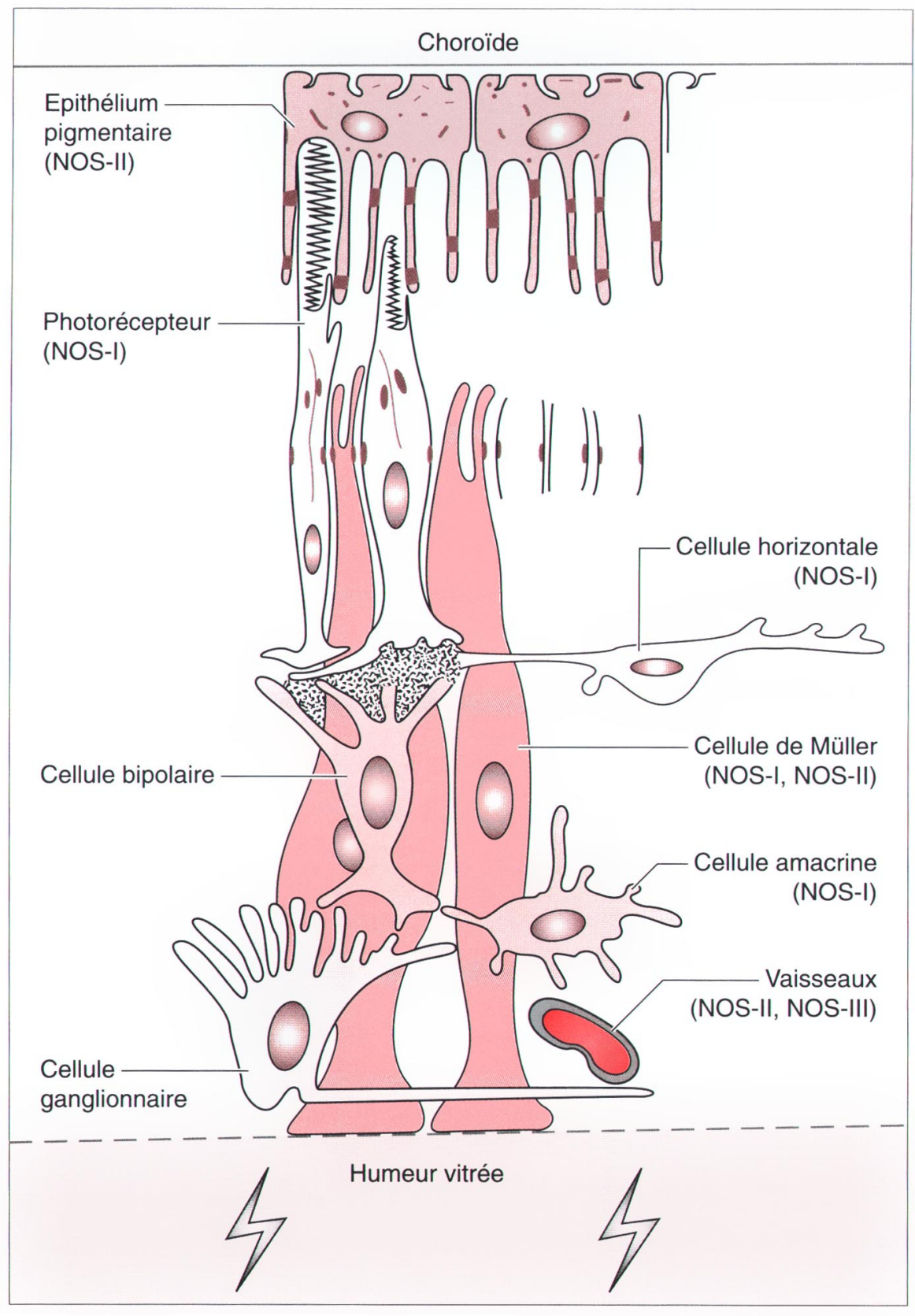

Figure 1. Coupe schématique des différentes cellules formant la rétine de mammifère avec la localisation des différentes isoformes de NOS. La NOS-I a été mise en évidence par immunohistochimie dans certaines cellules amacrines, dans les cellules gliales de Müller, dans des cellules horizontales et dans les photorécepteurs de différentes espèces. La NOS-Il est produite par les cellules de l'épithélium pigmentaire, les cellules gliales de Müller et dans la paroi des vaisseaux où elle a été récemment mise en évidence dans les cellules endothéliales vasculaires et dans les péricytes in vitro après stimulation par les cytokines et le LPS. Les cytokines et les facteurs de croissance jouent un rôle clé dans la régulation de sa synthèse. NOS-IIl semble n'être présente que dans la paroi des vaisseaux. veuses est actuellement bien établi dans l'artère ophthalmique et dans les artérioles rétiniennes [18, 19]. Cette production de $\mathrm{NO}$, contrôlant le tonus artériolaire, a pu être directement mesurée à la surface de la rétine en utilisant une micro-électrode sensible au NO insérée dans l'œil de mini-porc [20].

\section{NOS inductible (NOS-II)}

Dans la rétine, les cellules épithéliales pigmentées et les cellules gliales de Müller de différentes espèces (bovine, murine, humaine) synthétisent in vitro la NOS-II, lorsqu'elles sont stimulées par le lipopolysaccharide (LPS) et l'interféron $\gamma$ $(\mathrm{IFN} \gamma)$ [21-24]. Chez le rat et le bœuf, l'ARNm de NOS-II et l'activité enzymatique sont induits de façon synergique par la coopération du LPS, de l'IFN $\gamma$ et du tumor necrosis factor $\alpha$ (TNF $\alpha)$, alors que, chez l'homme, c'est la costimulation par IFN $\gamma$ et l'interleukine $1 \beta$ (IL-1 $\beta$ ) qui est indispensable (Tableau I).

Les cytokines et les facteurs de croissance sont des éléments clés dans la régulation de l'induction de NOS-II [2]. Dans le but de comprendre leur potentiel stimulateur ou inhibiteur, le rôle de certains de ces facteurs, présents dans la rétine, sur la production de NO dans les cellules épithéliales pigmentées et les cellules gliales de Müller a été recherché. Les résultats résumés dans le Tableau I montrent que les fibroblast growth factors (FGF) et le transforming growth factor $\beta$ (TGF $\beta$ ) ont des effets opposés sur la régulation de la production de NO par ces cellules. Concernant le mécanisme moléculaire mis en jeu, les mesures d'ARNm de NOS-II montrent que le FGF inhibe l'induction de la NOS dans les cellules épithéliales pigmentées de bouf au niveau transcriptionnel [25] alors que le TGF $\beta$ augmente l'accumulation du messager. Dans les cellules épithéliales pigmentées du rat et de l'homme, l'effet inhibiteur du TGF $\beta$ semble dû en partie à une diminution de l'ARNm de NOS-II. Ces résultats suggèrent que la régulation de la NOS dans chaque type de cellules pourrait dépendre des signaux de transduction activés par les cytokines et de leur éventuelle régulation croisée avec ceux des facteurs de croissance. 


\section{RÉFÉRENCES}

15. Ahmad I, Leinders-Zufall T, Kocsis JD, Shepherd GM, Zufall F, Barnstable CJ. Retinal ganglion cells express a cGMP-gated cation conductance activatable by nitric oxide donors. Neuron 1994; 12 : 155-65.

16. Kurenny DE, Moroz LL, Turner RW, Sharkey KA, Barnes S. Modulation of ion channels in rod photoreceptors by nitric oxide. Neuron 1994; 13 : 315-24.

17. Nöll GN, Billek M, Pietruck C, Schmidt KF. Inhibition of nitric oxide synthase alters light responses and dark voltage of amphibian photoreceptors. Neuropharmacology 1994; 33: 1407-12.

18. Haefliger IO, Flammer J, Lüscher TF. Nitric oxide and endothelin-1 are important regulators of human ophthalmic arteries. Invest Ophthalmol Vis Sci 1992; 33 : 23403 .

19. Toda N, Kitamura Y, Okamura T. Role of nitroxidergic nerve in dog retinal arterioles in vivo and arteries in vitro. Am J Physiol 1994; 35: H1985-92.

20. Donati G, Pournaras CJ, Munoz J, Poitry $S$, Poitry-Yamate CL, Tsacopoulos M. Nitric oxide controls arteriolar tone in the retina of the miniature pig. Invest Ophthalmol Vis Sci 1995 ; 36 : 2228-37.

21. Goureau O, Lepoivre M, Becquet F, Courtois Y. Differential regulation of inducible nitric oxide synthase by basic fibroblast growth factors and transforming growth factor $\beta$ in bovine retinal pigmented epithelial cells: inverse correlation with cellular proliferation. Proc Natl Acad Sci USA $1993 ; 90: 4276-80$.

22. Goureau O, Hicks D, Courtois Y. Human retinal pigmented epithelial cells produce nitric oxide in response to cytokines. Biochem Biophys Res Commun 1994; 198 : 1206.

23. Sparrow JR, Nathan CF, Vodovotz Y. Cytokine regulation of nitric oxide oxide synthase in mouse retinal pigment epithelial cells in culture. Exp Eye Res 1994; 59 : 12939 .

24. Goureau O, Hicks D, Courtois Y, de Kozak Y. Induction and regulation of nitric oxide synthase in retinal Müller glial cells. $J$ Neurochem 1994; $63: 310-7$.

25. Goureau O, Faure V, Courtois Y. Decrease of inducible nitric oxide synthase mRNA by fibroblast growth factors in bovine retinal pigmented epithelial cells. Eur J

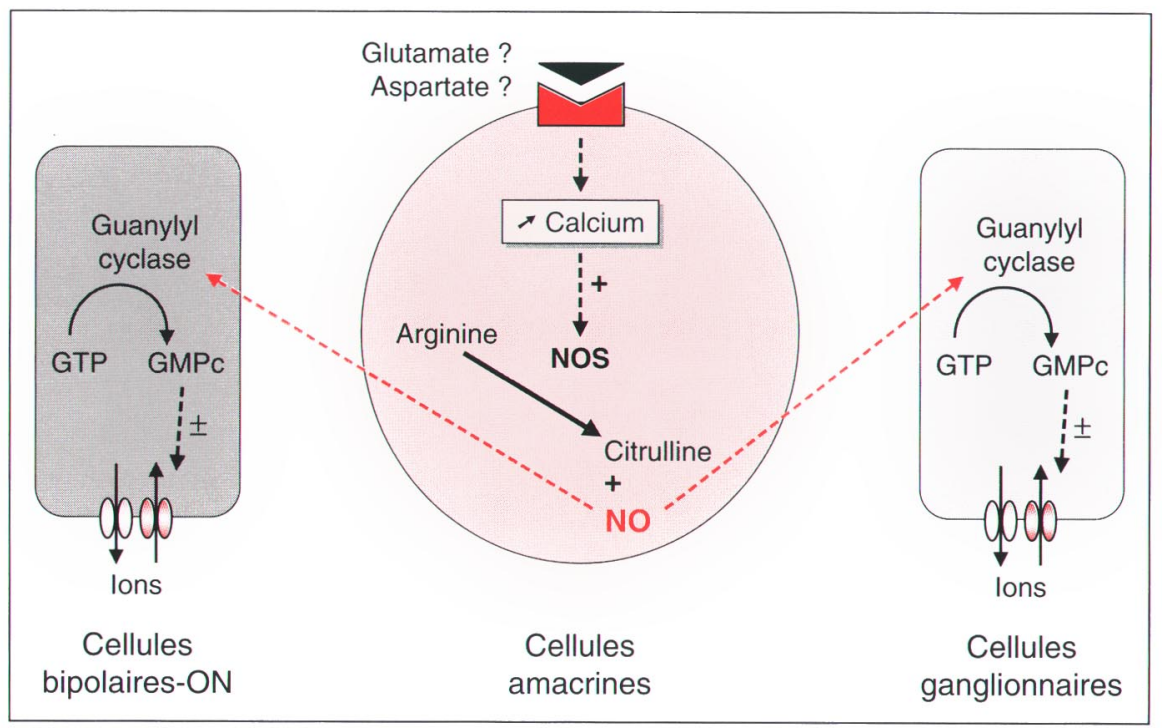

Figure 2. Mécanisme d'action potentiel du NO dans la rétine neurale. Le NO produit par les cellules amacrines, à la suite de l'activation du NOS par les neurotransmetteurs, pourrait diffuser et activer la guanylyl cyclase présente dans les cellules neuronales voisines, cellules bipolaires et ganglionnaires. Le GMPc ainsi produit pourrait moduler les mouvements ioniques dans ces cellules via son interaction directe ou indirecte (protéine kinase dépendante du GMPc) avec les canaux et ainsi moduler la transmission de l'influx.

Tableau I

EFFETS DIFFÉRENTIELS DES CYTOKINES ET DES FACTEURS DE CROISSANCE SUR L'INDUCTION DE NOS-II DANS LA RÉTINE

\begin{tabular}{|lcccc|}
\hline Type cellulaire & Cellules de Müller & \multicolumn{2}{c|}{ Cellules épithéliales pigmentées } \\
\hline Espèces & Rat & Souris/Rat & Bœuf & Homme \\
\hline Inducteurs & $\begin{array}{l}\text { LPS/IFN } \gamma \text { ou } \\
\text { LPS/IFN } \gamma / T N F \alpha\end{array}$ & LPS/IFN $\gamma / T N F \alpha$ & LPS/IFN $\gamma$ & IL1 $\beta /$ IFN $\gamma$ \\
\hline $\begin{array}{l}\text { FGF1/FGF2 } \\
\text { TGF } \beta\end{array}$ & - & $\begin{array}{c}\text { Potentialisation } \\
\text { Inhibition }\end{array}$ & $\begin{array}{c}\text { Inhibition } \\
\text { Potentialisation }\end{array}$ & $\begin{array}{c}\text { Potentialisation } \\
\text { Inhibition }\end{array}$ \\
\hline
\end{tabular}

Les effets d'une forte production de NO résultant de l'induction de NOS-II dans la rétine sont peu documentés. Un effet bénéfique, via ces propriétés antivirales, microbicides et antitumorales, semble probable. Cependant, il est possible qu'une forte libération de NO puisse perturber les processus de neurotransmission évoqués précédemment. Des expériences in vitro effectuées au laboratoire montrent que l'ajout d'un donneur de NO à des cellules épithéliales pigmentées diminue la capacité de ces cellules de phagocy- ter les segments externes des photorécepteurs [26]; cette inhibition n'est observée que si la production de NO a lieu durant le processus de phagocytose. Ce phénomène de phagocytose étant indispensable au bon fonctionnement de la rétine, on peut penser que si cette inhibition avait lieu in vivo de façon chronique, elle pourrait entraîner l'accumulation de débris de segments externes entre les photorécepteurs et l'épithéliales pigmentées, et conduire à une dégénérescence de ces photorécepteurs. 


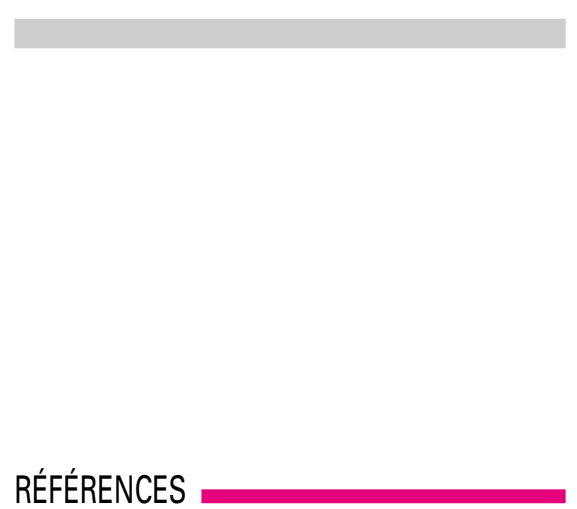

26. Becquet F, Courtois Y, Goureau O. Nitric oxide decreases in vitro phagocytosis of photoreceptor outer segments by bovine retinal pigmented epithelial cells. I Cell Physiol 1994 ; 159 : 256-62.

27. Digheiro P, Reux I, Hauw II, Fillet AM, Courtois Y, Goureau O. Expression of inducible nitric oxide synthase in cytomegalovirus-infected glial cells of retinas from AIDS patients. Neurosci Lett 1994; 166: 31-4.

28. Davidson PC, Sterneberg P Jr. Potential retinal phototoxicity. Am J Ophthalmol 1993; 116: 497-501.

29. Goureau O, Bellot J, Thillaye B, Courtois Y, de Kozak Y. Increased nitric oxide production in endotoxin-uveitis: reduction of uveitis by an inhibitor of nitric oxide synthase. J Immunol 1995; 154: 6518-23.

30. Goureau O, Jeanny JC, Becquet F, Harthmann MP, Courtois Y. Protection against light-induced retinal degeneration by an inhibitor of NO synthase inhibitor. Neuro Report 1993 ; 5 : 233-6.

31. Dawson DA. Nitric oxide and focal ischemia: multiplicity of actions and diverse outcome. Cerebrovasc Brain Metab Rev 1994; $6: 299-324$

32. Geyer O, Almog J, Lupu-Meiri M, Lazar M, Oron Y. Nitric oxide synthase inhibitors protect rat retina against ischemic injury. FEBS Lett 1995; 374: 399-402.

33. Ostwald P, Goldstein IM, Pachnanda A, Roth S. Effect of nitric oxide synthase inhibition on blood flow after retinal ischemia. Invest Ophthalmol Vis Sci 1995; 36 : 2396-403.

34. Nathanson JA, McKee Mc. Alterations of ocular nitric oxide synthase in human glaucoma. Invest Ophthalmol Vis Sci 1995; 36 : 1774-84.

35. Stamler S. Redox signaling: nitrosylation and related target interactions of nitric oxide. Cell 1994; 78: 931-6.

36. Koppenol WH, Moreno JJ, Pryor WA, Ischiropoulos H, Beckman IS. Peroxynitrite, a cloaked oxidant formed by nitric oxide and superoxide. Chem Res Toxicol 1992; 5 : 834-42.

37. Cazevieille C, Muller A, Meynier F, Bonne C. Superoxide and nitric oxide cooperation in hypoxia/reoxygenation induced neuron injury. Free Radic Biol Med 1993; 14 : 389-95.

$\mathrm{m} / \mathrm{s} n^{\circ} 5$, vol. 12 , mai 96

\section{Expression possible de la NOS inductible (NOS-II) dans les différentes affections de la rétine} NO et rétinites

Des études d'immunohistochimie réalisées au laboratoire ont révélé que la NOS de type II est produite dans les rétines de patients atteints de rétinite à cytomégalovirus (CMV) au cours du SIDA, alors qu'elle ne l'est pas dans les rétines de sujets sains [27]. Les expériences de double marquage en immunohistochimie ont permis de localiser la NOS-II dans les cellules gliales (cellules de Müller et astrocytes) de la rétine, préalablement infectées par le CMV. L'IFN $\gamma$, dont les concentrations sont très élevées dans l'œil des patients séropositifs atteints de rétinite à CMV, pourrait être un candidat pour l'induction de la NOS-II dans cette affection. Le rôle bénéfique (antiviral) ou néfaste (dégradation tissulaire) du NO au cours des rétinites à CMV reste à établir.

Plus récemment, l'induction de NOSII dans la rétine et la production consécutive de nitrite (métabolite final stable du NO) dans le vitré ont pu être mises en évidence au cours d'une maladie inflammatoire murine, l'uvéite induite par endotoxine, qui représente un bon modèle d'inflammation oculaire (uvéite) chez l'homme [28]. Par ailleurs, l'injection intrapéritonéale d'inhibiteur de NOS à ces animaux prévient la production de NO dans l'œil et diminue fortement la sévérité de la maladie [28], démontrant l'implication du NO dans le développement de cette maladie et laissant entrevoir d'importantes possibilités thérapeutiques. L'utilisation plus locale (collyre, iontophorèse) d'inhibiteurs sélectifs de la NOS-II tels que l'aminoguanidine est actuellement entreprise dans ce modèle d'uvéite induite par endotoxine chez le rat.

\section{NO et dégénérescence rétinienne} photo-induite

Depuis longtemps, il a été proposé que la production de radicaux libres oxygénés (RLO) par la lumière pouvait en partie rendre compte de la dégénérescence des photorécepteurs induite par une forte illumination [29]. Au cours de l'illumination, l'épaisseur de la couche des noyaux des photorécepteurs diminue de façon progressive avec l'éclairement pour atteindre une épaisseur minimale après cinq jours d'illumination. Chez les animaux traités, tous les deux jours, par un inhibiteur de la NOS par voie intrapéritonéale, une protection significative a été observée tout au long de la rétine [30]. L'analyse de l'expression des ARNm de la NOS-II par RT-PCR montre que cette isoforme est induite au cours de l'éclairement dans des extraits de rétine neurale et du complexe épithélium pigmentaire/choroïde et que l'intensité du message semble proportionnelle aux dommages induits par la lumière. Le mode d'action de NO dans ce processus de dégénérescence reste à déterminer (augmentation accrue du GMP cyclique, nitrosylation de certaines protéines de la transduction visuelle, inhibition de la phagocytose des photorécepteurs par les cellules épithéliales pigmentées).

Dans les phénomènes d'ischémie cérébrale l'implication du NO est bien établie, même si son rôle exact (protecteur ou destructeur) commence à peine à être compris [31] En revanche, la participation de NO dans les ischémies rétiniennes est encore peu étudiée [32, 33], mais on peut penser que les résultats seront très proches de ceux obtenus dans le cerveau.

Il convient également de noter qu'une dysrégulation de la production de NO par les NOS-I et III, dites constitutives, pourrait entraîner un dysfonctionnement, voire une dégénérescence de la rétine, par exemple par l'intermédiaire d'une mauvaise régulation du débit sanguin dans la rétine. Aucun résultat dans ce sens n'a encore été rapporté dans la littérature, même si, dans le segment antérieur de l'œil, l'absence de NOSIII dans les fibres musculaires du trabeculum pourrait rendre compte d'une augmentation de la pression intra-oculaire chez les patients atteints de glaucome [34] et entrâ̂ner ainsi à long terme une dégénérescence de la rétine.

En résumé, la rétine est un modèle particulièrement intéressant dans lequel les trois isoformes de la NOS 
ont pu être identifiées. Un point important serait aujourd'hui d'essayer d'attribuer un ou des rôles précis à chacune des trois isoformes et d'étudier la possibilité de régler spécifiquement leur synthèse ou leur activité grâce à des inhibiteurs spécifiques. Dans ce contexte, une étude approfondie de la physiologie et des affections de la rétine serait intéressante dans les modèles d'invalidation des gènes de chacune des trois isoformes de la NOS. Par ailleurs, la compréhension de la chimie du NO et l'étude in vivo du métabolisme du NO dans l'œil s'avèrent totalement indispensables, puisque la toxicité de NO semble dépendre non seulement de l'origine de sa synthèse (forme inductible ou constitutive) mais également de sa forme chimique (NO radical, $\mathrm{NO}^{+}$ou $\mathrm{NO}^{-}$), qui est elle-même fonction de l'environnement redox dans lequel NO est synthétisé [35]. Dans ce contexte, nous avons pu montrer que l'injection intra-oculaire aiguë de donneurs de NO n'induit pas de réaction inflammatoire ou toxique au niveau de la rétine, suggérant que le NO à lui seul n'est pas capable d'induire des lésions tissulaires dans l'œil. Ainsi les effets toxiques du NO observés dans les différentes maladies (uvéite, photodégénérescence...) pourraient être dus à la formation de peroxynitrite, via l'interaction du NO avec les radicaux libres oxygénés [36, 37], qui peuvent être produits dans ces maladies oculaires

\section{Summary}

Nitric oxide in the retina: a double face mediator

The free radical nitric oxide $(\mathrm{NO})$ is synthetized from L-arginine by an enzyme termed NO synthase (NOS). Three isoforms of NOS, coded by three disctint genes, have been identified, NOS-I and NOSIII, termed constitutive isoforms and NOS-II, generally termed as inducible isoform. The three isoforms are expressed in the retina and the specific role for each of them begin to be understood. The small quantities generate by isoforms I and III in a regulated manner, is involved in neurotransmission between some amacrine cells and bipolar or ganglion cells, and in the regulation of retinal blood flow. In contrast, NOS-II is expressed in retinal pigmented epithelial and Müller glial cells, during some pathological status or after in vitro stimulation by cytokines. The large amounts of NO, released by NOSII, can act as a cytostatic and cytotoxic molecule against invading agents but also against healthy cells. In this context the enhanced formation of $\mathrm{NO}$ could disrupt the functional retinal integrity, could lead to retinal inflammation and then could participate to retinal degeneration. 